\title{
AVALIAÇÃO DOS NÍVEIS DE VIBRAÇÃO VERTICAL NO ASSENTO DE UM TRATOR AGRÍCOLA DE PNEUS UTILIZANDO UM SISTEMA DE AQUISIÇÃO AUTOMÁTICA DE DADOS ${ }^{1}$
}

\author{
Paulo Fernando dos Santos Filho², Haroldo Carlos Fernandes ${ }^{3}$, Daniel Marçal de Queiroz ${ }^{3}$, Amaury Paulo de \\ Souza ${ }^{4}$ e Arlindo José Camilo 5
}

\begin{abstract}
RESUMO - Este trabalho foi realizado na Faculdade de Engenharia de Guaratinguetá (FEG-UNESP), SP, no laboratório de vibração e acústica em uma área experimental, com os objetivos de: a) determinar os níveis de vibração vertical no assento e na base do assento de um trator agrícola, executando a operação de gradagem para diferentes velocidades de trabalho; b) caracterizar a faixa de freqüência que apresenta os maiores picos de vibração vertical no assento do operador do trator; e c) analisar o conforto do operador, comparando os níveis de vibração obtidos com as principais normas vigentes. O sistema de aquisição de dados foi constituído por sensores de vibração, por condicionadores e amplificadores e por um conversor analógico-digital, instalados em um microcomputador e embarcados no trator ensaiado. Posteriormente os dados foram tratados pelo programa ORIGIN 50. Com base nos resultados obtidos, concluiu-se que: os níveis de aceleração encontrados na parte superior do assento foram consideravelmente inferiores aos níveis da base para as três velocidades, sendo a terceira marcha a que apresentou os maiores níveis de vibração; a faixa de freqüência que apresentou os maiores picos de vibração vertical foi entre 2 e $4 \mathrm{~Hz}$; os valores de aceleração ponderada global encontraram-se bem acima dos limites definidos pela norma ISO 2631 para um período de 8 horas de trabalho; e houve crescimento dos níveis de vibração com o aumento da velocidade do sistema trator-implemento.
\end{abstract}

Palavras-chave: Vibração, sensores e trator.

\section{EVALUATION OF THE VERTICAL VIBRATION LEVELS IN A TIRE TRACTOR SEAT BY APPLYING AN AUTOMATIC DATA AQUISITION SYSTEM}

\begin{abstract}
A study was carried out at the Faculdade de Engenharia de Guaratinguetá - FEG-UNESP, SP, in the acoustic and vibration laboratory as well as in an experimental area, to: a) determine the levels of vertical vibration in both the seat and seat base of an agricultural tire tractor under operation (harrowing) at different forward speeds; $b$ ) characterize the frequency range presenting the highest vertical vibration peaks in the tractor operator seat; c) analyze ride comfort for tractor operators by comparing the actual level of vibration with that of the main effective norms. The data acquisition system consisted of some vibration sensors, conditioners, amplifiers, and a digital-analogical converter plugged to a microcomputer and connected to the tested tractor. Later, the data were treated by the program ORIGIN 50. Based on the results, it was concluded that the acceleration levels obtained at the upper part of the seat were much lower than those in the seat base at the three operational speeds, whereas the third gear presented the highest vibration levels; the frequency range from 2 to $4 \mathrm{~Hz}$ presented the highest peaks of vertical vibration; the values of the total weighted acceleration were found to be much above the limits determined by the norm ISO 2631 for an 8-working hour period; the vibration levels increased as the forward speed of the tractor-implement system increased.
\end{abstract}

Key works: Vibration, sensors and tractor.

1 Recebido para publicação em 19.9.2002.

Aceito para publicação em 9.9.2003.

Parte da dissertação de mestrado do 1o autor.

2 Doutorando em Engenharia Agrícola, Universidade Federal de Viçosa - UFV, 36570-000 Viçosa-MG; ${ }^{3}$ Prof. Adjunto do Departamento de Engenharia Agrícola - UFV, <haroldo@ufv.br>; ${ }^{4}$ Prof. Titular do Departamento de Engenharia Florestal UFV; ${ }^{5}$ Estudante de graduação do curso de Engenharia Agrícola e Ambiental - UFV. 


\section{INTRODUÇÃOO}

O avanço das pesquisas em mecanização agrícola levou à substituição da tração animal por máquinas modernas que têm resolvido uma série de problemas de plantio e colheita. No entanto, o número crescente de operadores sem treinamento correto de segurança e higiene no trabalho fez com que o número de acidentes no campo aumentasse vertiginosamente.

Os tratores agrícolas em circulação no País, na sua maioria, apresentam problemas de conforto e segurança para os operadores, uma vez que estes ficam expostos a níveis de insalubridade acima do permitido pelas normas de segurança do trabalho (Mathias, 1989).

Os tratores e as máquinas agrícolas, em geral, produzem vibrações de baixa freqüência que são transmitidas para o posto do operador. Estas freqüências podem gerar problemas de visão, irritabilidade, deformações lombares e problemas digestivos. Para amortecer e amenizar estas vibrações de baixa freqüência, têm-se estudado várias alternativas de implantação de um sistema de suspensão mais eficiente para o posto do operador.

Schlosser \& Debiasi (2002) relataram que os conhecimentos em ergonomia resultaram em novos conceitos e que estes fizeram com que os fabricantes passassem a oferecer modelos de tratores com maior conforto para localização de comandos e instrumentos.

Mehta \& Tewari (2000) descreveram as várias maneiras de analisar o conforto do assento do trator, tanto de forma objetiva como subjetiva. Para a análise objetiva foram relatados os tipos de sensores que avaliam a pressão de contato do corpo do operador com o assento, exposto a várias freqüências de vibração. Concluiu-se que para fazer um estudo completo de conforto deve-se levar em consideração informações específicas de biomecânica, níveis de vibração, distribuição de pressão no assento, postura do operador e material do coxim.

Robin (1987), citado por Nagaoka (2001), relatou que as pesquisas realizadas com tratores agrícolas com e sem lastros e de diferentes massas indicaram que as vibrações com freqüências compreendidas no intervalo de $2 \mathrm{a}$ $4 \mathrm{~Hz}$ apresentam os maiores picos.

Na prática, as vibrações consistem em uma mistura complexa de diversas ondas, com freqüências e direções diferentes. A partir da análise desses componentes é possível calcular o nível médio das vibrações, que pode

R. Árvore, Viçosa-MG, v.27, n.6, p.887-895, 2003 ser usado para estimar o impacto dessas ondas no corpo humano (Dul \& Weerdmeester,1995).

A exposição de trabalhadores à vibração na colheita florestal pode ser parcial ou total, conforme as partes do corpo em contato com as fontes. Analisando sob o ponto de vista da medicina do trabalho, Smith \& Thomas Junior (1993) citam que a exposição parcial mais perigosa é a das mãos e dos braços dos trabalhadores, causada por ferramentas motorizadas, como a motoserra. As vibrações totais estão presentes nos trabalhadoes florestais e agrícolas.

Schlosser \& Debiasi (2002) afirmaram que a coluna vertebral dos operadores de máquinas é uma das partes mais atingidas pelas doenças ocupacionais oriundas da operação de tratores agrícolas. De acordo com os autores nos Estados Unidos, o NATIONAL SAFETY COUNCIL diagnosticou que havia no país 400.000 lesões de coluna, ocasionadas pelo trabalho, que incapacitam o acidentado para o trabalho.

Mathias (1989) analisou o conforto do sistema trator-carreta, utilizando as curvas da norma ISO-2631. As medidas de vibrações foram obtidas experimentalmente no assento do tratorista. Da análise dos dados constatouse que o tempo necessário para que as atividades do tratorista não sejam prejudicadas pela fadiga é limitado em 7 horas.

Um trabalho realizado por Pessina (1986), citado por Berasategui (2000), teve por objetivo relacionar as vibrações sobre os assentos de tratores agrícolas, durante a fase de transporte, com a velocidade de deslocamento e o engate de implementos. O trator deslocou-se sobre uma pista agrícola acidentada e uma normalizada para ensaios de assento, constituída de tábuas de madeiras, normalmente chamada de pista ISO 5007. As velocidades utilizadas no experimento foram $12,15,17$ e $20 \mathrm{~km} / \mathrm{h}$. Nesse trabalho concluiu-se que:

- o nível de aceleração média eficaz cresceu com o aumento da velocidade de deslocamento, sobretudo na direção vertical;

- as acelerações verticais no assento do tratorista predominaram sobre as longitudinais e laterais;

- os implementos de arrasto geraram níveis maiores de aceleração no assento do tratorista do que os implementos suspendidos; e

- a freqüência dominante do trator encontrou-se em $2,5 \mathrm{~Hz}$. 
No Brasil, a Associação Brasileira de Normas Técnicas (ABNT) desenvolveu a NBR 12319 (1995), a respeito de medição da vibração transmitida ao operador de tratores agrícolas de rodas e máquinas agrícolas. Esta norma fixa, além dos métodos para medir e registrar a vibração do corpo humano, as condições de operação da máquina e as características da pista artificial opcional de ensaios.

Considerando que a vibração de um trator é geralmente mais intensa que a dos demais ambientes em que ele possa permanecer e levando em consideração as exposições anteriores, os principais objetivos deste trabalho foram:

- Determinar os níveis de vibração vertical no assento e na base do assento de um trator agrícola, executando a operação de gradagem para diferentes velocidades de trabalho.

- Caracterizar a faixa de frequiência que apresenta os maiores picos de vibração vertical no assento do operador do trator.

- Analisar o conforto do operador, comparando os níveis de vibração obtidos com os previstos pelas principais normas vigentes.

\section{MATERIAL E MÉTODOS}

\subsection{Localização do Experimento}

O experimento foi conduzido no município de Guaratinguetá-SP, cuja altitude média é de $537 \mathrm{~m}$, a longitude de $22^{\circ} 52^{\prime} 40^{\prime \prime} \mathrm{W}(\mathrm{Gr})$ e a latitude de $22^{\circ} 48^{\prime} 43^{\prime \prime} \mathrm{S}$.

\subsection{Trator}

O trator encontrava-se lastrado, com o tanque, o radiador e os reservatórios de fluídos de lubrificação e hidráulicos cheios. Os pneus utilizados no ensaio eram de tamanho padronizado para o trator, conforme especificação do fabricante. A profundidade das garras estavam de acordo com a NBR 12319 (1992), segundo a qual a profundidade não deve ser menor do que $65 \%$ da profundidade das garras dos pneus novos.

\section{Características do trator}

- Marca/modelo: Perkins 4000.

- Ano de fabricação: 1999.

- Tipo: Diesel, injeção direta, quatro-tempos.
- Cilindros: 4, verticais em linha.

- Cilindrada: $4000 \mathrm{~cm}^{3}$.

- Relação de compressão: 16:1

- Potência no motor a $2.200 \mathrm{rpm}: 55,2 \mathrm{~kW}(75 \mathrm{cv})$.

- Tração dianteira auxiliar.

- Rodagem diagonal: Rodagem dianteira 12,4-24 R1, com pressão de insuflação de $96,5 \mathrm{kPa}$ (14psi); traseira 18,4-30 R1 com pressão de $110,3 \mathrm{kPa}$ (16 psi), em boas condições.

- Massa sem lastro: $2553 \mathrm{~kg}$.

- Massa com lastro: $3673 \mathrm{~kg}$.

- Sem cabine e estrutura de proteção contra capotamento, com toldo.

\subsection{Implemento}

O implemento utilizado foi uma grade destorroadora-niveladora off-set, com 28 discos de $0,5 \mathrm{~m} \mathrm{x}$ $0,0035 \mathrm{~m}$ (20"x $3,5 \mathrm{~mm})$ e massa aproximada de $666 \mathrm{~kg}$. A largura de trabalho foi 2,6 m.

\subsection{Caracterização da Área}

O solo foi classificado como Latossolo VermelhoAmarelo de textura franco-argilosa, de acordo com a classificação estabelecida pela EMBRAPA (1999). Antes da gradagem, foi realizada uma aração com um arado de discos de 3 x 26", à profundidade de $0,18 \mathrm{~m}$. A gradagem foi realizada no sentido longitudinal da aração.

\subsection{Características do Operador}

A massa do operador do trator foi $69,4 \mathrm{~kg}$, valor este que se encontra na faixa entre $65 \pm 5 \mathrm{~kg}$, recomendada pela NBR 12319 (1992). A massa do operador foi registrada, pois poderia interferir nas medições de vibração.

\subsection{Velocidade de Deslocamento}

As velocidades utilizadas nos testes foram $1,39 \mathrm{~m} / \mathrm{s}, 1,67 \mathrm{~m} / \mathrm{s}$ e $1,95 \mathrm{~m} / \mathrm{s}$, para $1^{\mathrm{a}}, 2^{\mathrm{a}}$ e $3^{\mathrm{a}}$ marchas, respectivamente. A velocidade real foi determinada, medindo-se, com um cronômetro digital, o tempo gasto para percorrer $30 \mathrm{~m}$. A rotação do motor do trator foi de $1.800 \mathrm{rpm}$, em todos testes.

R. Árvore, Viçosa-MG, v.27, n.6, p.887-895, 2003 


\subsection{Montagem do Experimento}

As medições de vibração foram realizadas para três diferentes velocidades do conjunto trator-implemento, tendo sido realizada uma passada com o tempo total de 60 segundos para cada velocidade. A frequiência de aquisição foi de 400 pontos por segundo, gerando 24.000 pontos de vibração e ruído, para cada velocidade. Para análise estatística dos sinais de vibração, considerou-se a distribuição de seu valor médio quadrático para cada frequiência que o sinal contém, ou seja, a análise espectral de potência.

O sistema trator-implemento foi testado sob condições de campo, em uma área plana, sem obstáculos e declives, pois estes poderiam ocasionar amplitudes vibratórias anômalas.

A grandeza primária usada para descrever a intensidade da vibração foi a aceleração, expressa $\mathrm{em} \mathrm{m} / \mathrm{s}^{2}$. As determinações dos níveis de vibração foram baseadas nas normas ISO 2631 (1997) e NBR 12319 (1992) e na metodologia de Berasategui (2000) e Mathias (1989).

Para análise do domínio da freqüência, foram utilizados o programa ORIGIN 50 e o programa SIMASPC. O SIMAS-PC é um programa de manipulação e análise de sinais, que faz interface com a placa CAD10/ 26 utilizada na aquisição de dados.

Para análise da aceleração ponderada eficaz utilizaram-se filtros digitais, segundo a metodologia de Berasategui (2000). Os sinais foram transformados para o domínio da freqüência, utilizando-se a função FFT amplitude e fase, sendo filtrados digitalmente em bandas de $1 / 3$ de oitava para obtenção da aceleração eficaz. Os valores máximos obtidos, em cada banda, foram multiplicados por fatores de ponderação, gerando as acelerações ponderadas. Para análise da aceleração eficaz ponderada global na faixa entre 1 e $80 \mathrm{~Hz}$, foi utilizada a NBR 12319 (1992), sendo a mesma determinada por meio da equação

$$
a_{w}=\sqrt{\sum_{f} w_{f}{ }^{2} b_{f}{ }^{2}}
$$

em que $a_{w}=$ aceleração eficaz ponderada global; $w_{f}=$ fator de ponderação correspondente à frequiência $f$ em bandas de $1 / 3$ de oitava; e $b_{f}=$ valor da aceleração eficaz correspondente a freqüência $f$ em bandas de 1/3 de oitava.

R. Árvore, Viçosa-MG, v.27, n.6, p.887-895, 2003

\subsection{Equipamentos para Medição dos Níveis de Vibração}

Para coleta dos sinais de vibração, foram instalados dois acelerômetros piezoelétricos, um no assento do tratorista e outro na base do assento. Estes acelerômetros foram do tipo 91091 e 2323 da VEB-ROBOTRON. Para transformar volts em $\mathrm{m} / \mathrm{s}^{2}$, utilizaram-se as relações $2,36 \mathrm{mV} / \mathrm{m} / \mathrm{s}^{2}$ do acelerômetro 91091 e $2,07 \mathrm{mV} / \mathrm{m} / \mathrm{s}^{2}$ do acelerômetro 2323, sendo os dados extraídos das cartas dos sensores. Para caracterizar o ganho do amplificador, foi utilizada uma mesa calibradora da VEBROBOTRON, com o sinal conhecido de aceleração e frequiência.

Os sinais analógicos de saída do sensor foram condicionados e amplificados por um circuito eletrônico (HUMAN-RESPONSE VIBRATION METER robotron M 1300) e, posteriormente, enviados para um conversor analógico-digital (modelo LYNX CAD 10/26; 12 bits; $+10 \mathrm{~V}$ e $-10 \mathrm{~V}$ e taxa de conversão A/D $40 \mathrm{kHz}$ ), usado como interface com um computador PC-XT. Os equipamentos foram alimentados por uma bateria de $12 \mathrm{~V}$, ligada a um inversor de tensão (12V-DC/ 110V- AC).

\section{RESULTADOS E DISCUSSÃO}

\subsection{Medições dos Níveis de Vibração Vertical no Assento e na Base do Assento}

As Figuras 1, 2 e 3 apresentam os valores dos níveis vibratórios no assento, obtidos experimentalmente. Podese verificar que o sistema de suspensão do banco reduziu, sensivelmente, o nível de aceleração ao qual o operador foi submetido. Para que a análise dos dados fosse mais objetiva, segundo a metodologia de Mathias (1989), considerou-se parte do teste de 60 segundos. Na terceira marcha, foram encontradas as maiores acelerações instantâneas, observando-se valores de até $20 \mathrm{~m} / \mathrm{s}^{2}$ na base do assento, enquanto no assento o máximo foi de $2,5 \mathrm{~m} / \mathrm{s}^{2}$. Os valores altos de aceleração vertical são devido à movimentação do trator sobre uma superfície arada e ao implemento tracionado.

\subsection{Faixa de Freqüência dos Maiores Picos de Vibração no Assento do Operador}

A Figura 4 apresenta a análise com base na densidade espectral de potência PSD em $\left(\mathrm{m} / \mathrm{s}^{2}\right)^{2} / \mathrm{Hz}$ dos níveis de aceleração, para as três marchas ensaiadas. A faixa 
de frequiência de 2 a $4 \mathrm{~Hz}$ apresentou os maiores picos de vibração vertical no assento do trator, para operação de gradagem, o que constitui uma preocupação para os tratoristas, considerando que o valor encontrado está na faixa de frequiência de ressonância que afeta a coluna vertebral.
Robin (1987), citado por Nagaoka (2001), relatou que as pesquisas realizadas com tratores agrícolas com e sem lastros e de diferentes massas indicaram que as vibrações com frequiências compreendidas no intervalo de 2 a $4 \mathrm{~Hz}$ apresentam os maiores picos.

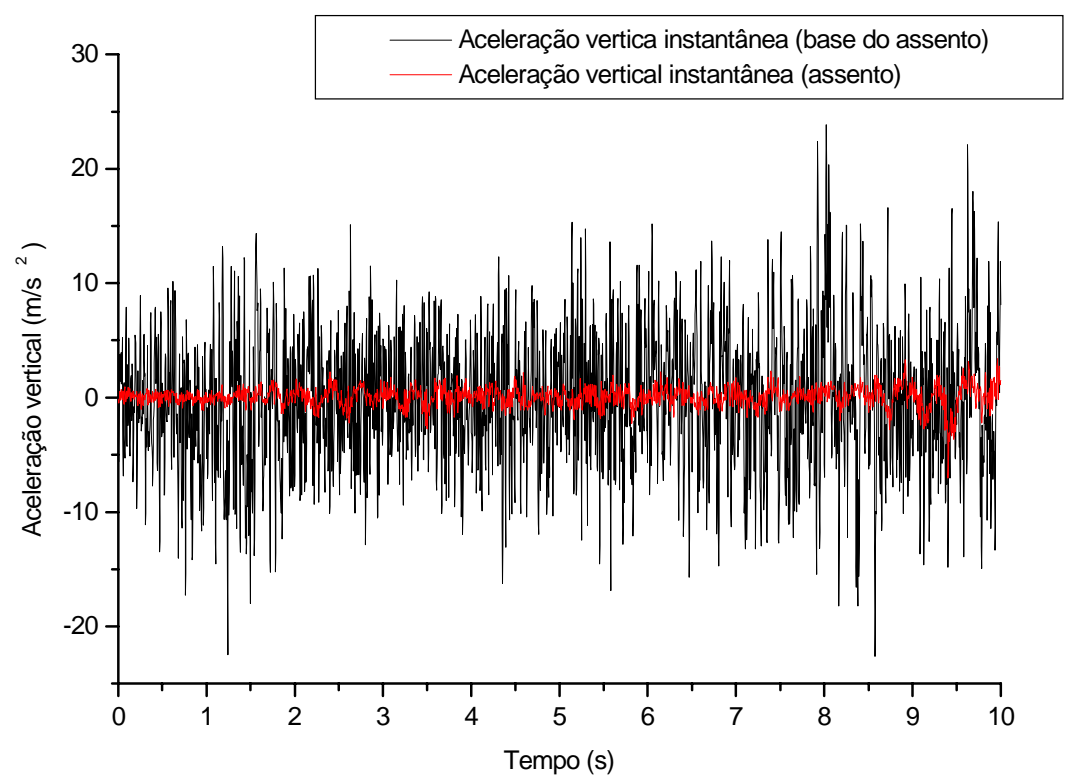

Figura 1 - Aceleração vertical instantânea nas partes superior e inferior do assento (terceira marcha). Figure 1 - Instantaneous vertical acceleration in the superior and inferior parts of the seat (third gear).

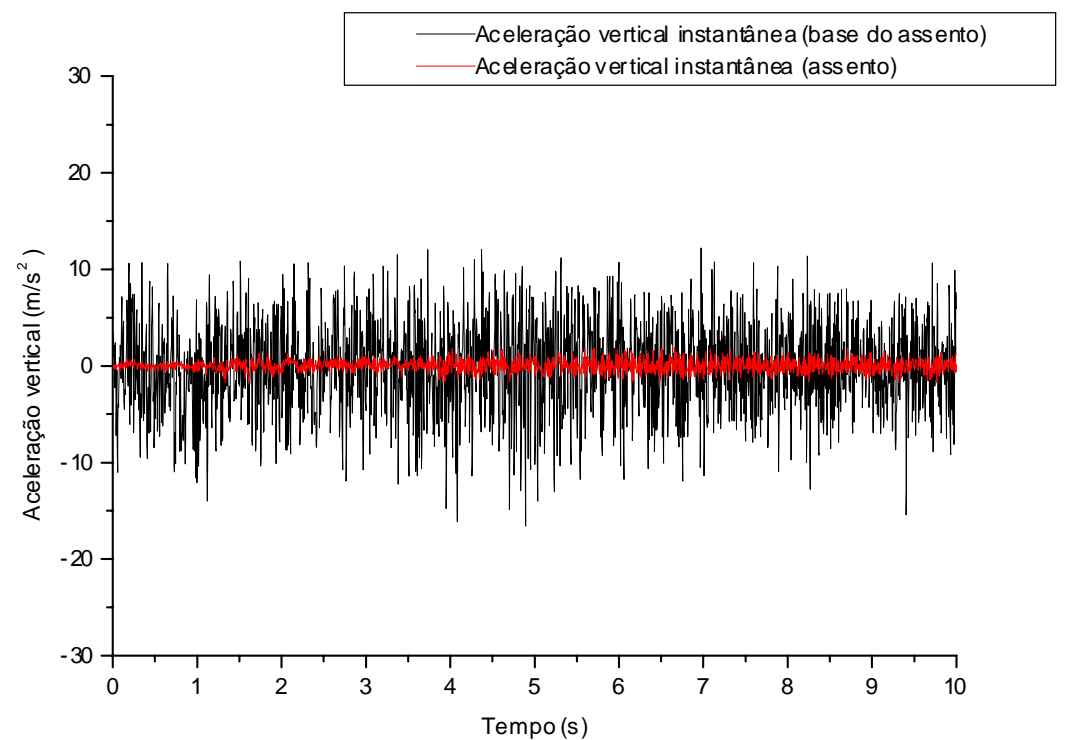

Figura 2 - Aceleração vertical instantânea na parte superior e inferior do assento (segunda marcha).

Figure 2 - Instantaneous vertical acceleration in the superior and inferior parts of the seat (second gear). 


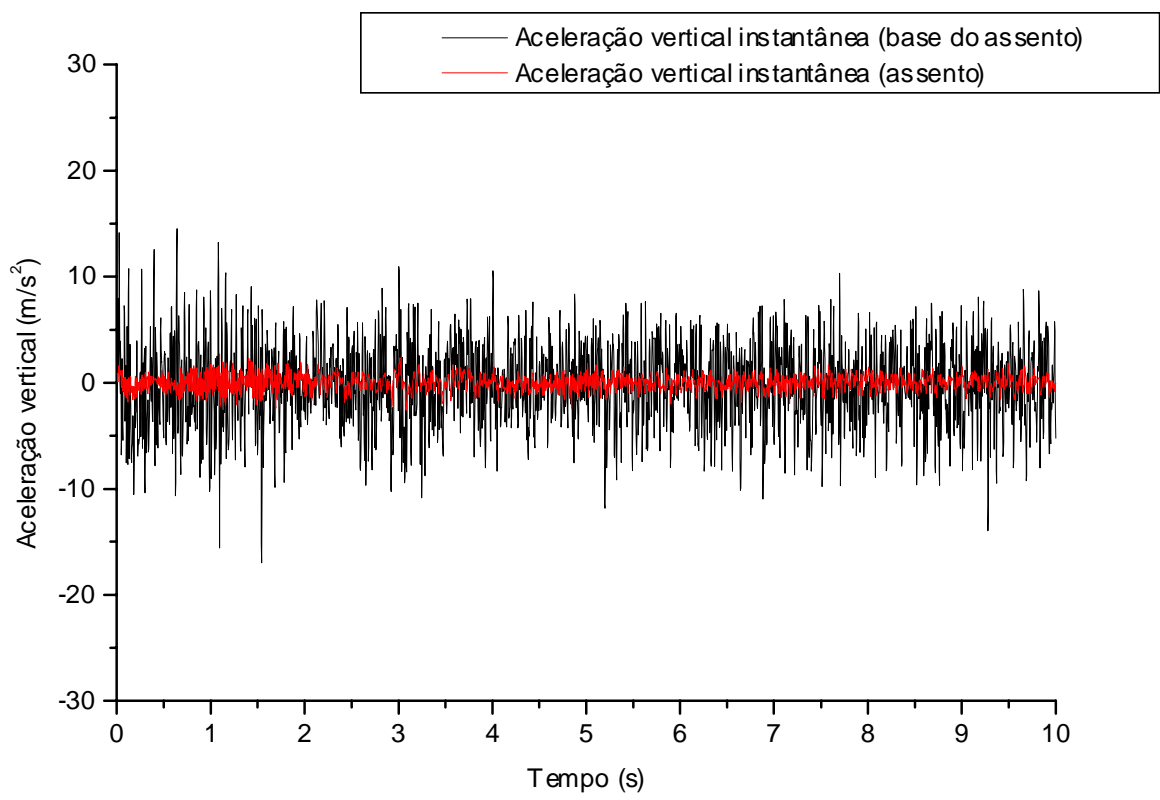

Figura 3 - Aceleração vertical instantânea nas partes superior e inferior do assento (primeira marcha). Figure 3 - Instantaneous vertical acceleration in the superior and inferior parts of the seat (first gear).

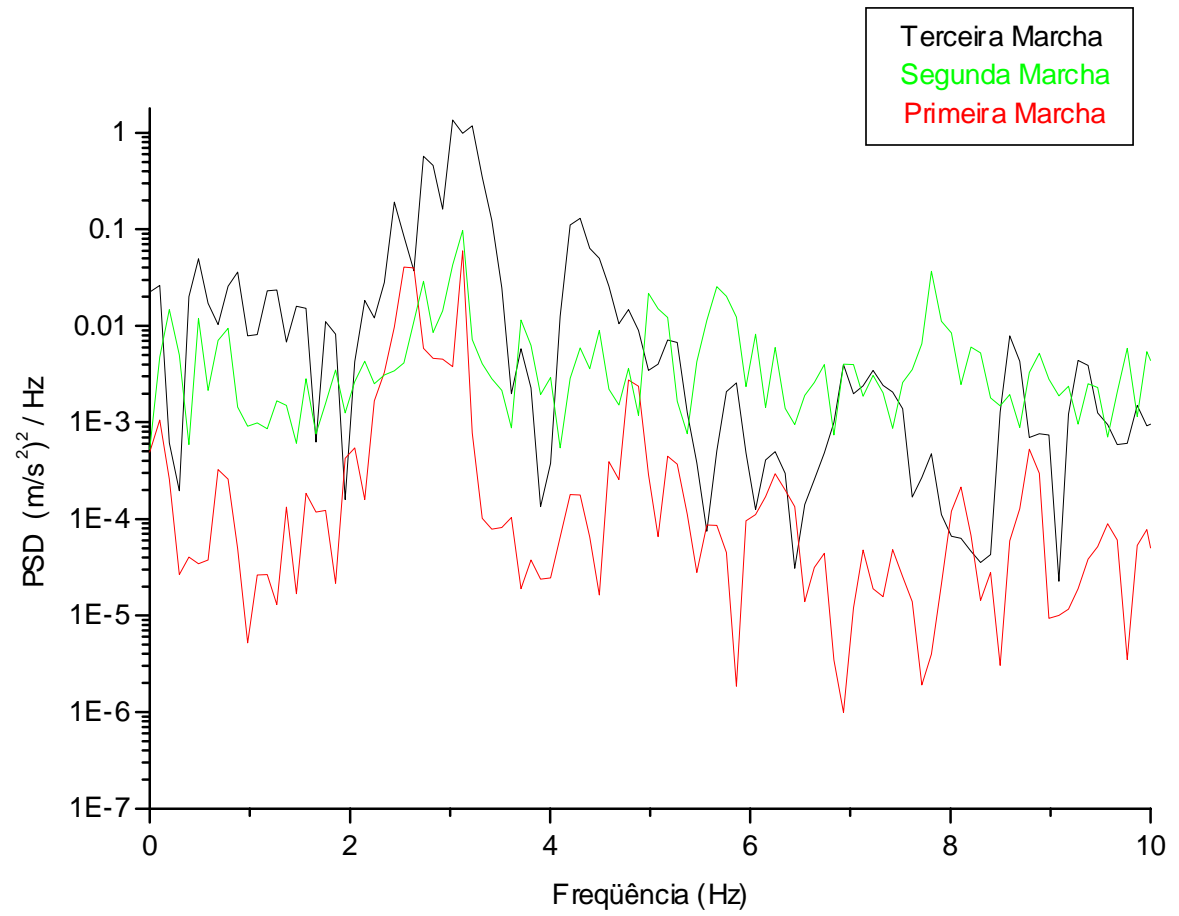

Figura 4 - Densidade espectral de potência para três marchas em estudo.

Figure 4-Spectral potency density for the three gears in the study.

R. Árvore, Viçosa-MG, v.27, n.6, p.887-895, 2003 
Morrison \& Harrington (1961), citados por Barger et al. (1963), verificaram que a freqüência real de um trator independe da velocidade de deslocamento e que a freqüência predominante está em torno de $3 \mathrm{~Hz}$. Segundo os autores, normalmente em um estudo de distribuição de freqüência a freqüência predominante será mais ou menos igual à frequiência natural.

Para que o tratorista possa usufruir conforto dinâmico, ele deverá estar isolado, ou suspenso em um assento que tenha uma frequiência natural diferente da freqüência natural do trator (Barger et al., 1963).

\subsection{Comparação dos Níveis de Vibração Encontrados Durante a Gradagem com os Valores Estabele- cidos pelas Principais Normas Técnicas}

A Figura 5 apresenta os valores de aceleração eficaz em bandas de $1 / 3$ de oitava. Os valores de aceleração ponderada em bandas de $1 / 3$ de oitava estão apresentados na Figura 6, enquanto a Figura 7 apresenta o gráfico da relação entre a aceleração eficaz ponderada global e a velocidade de deslocamento do trator, com a respectiva linha de tendência.

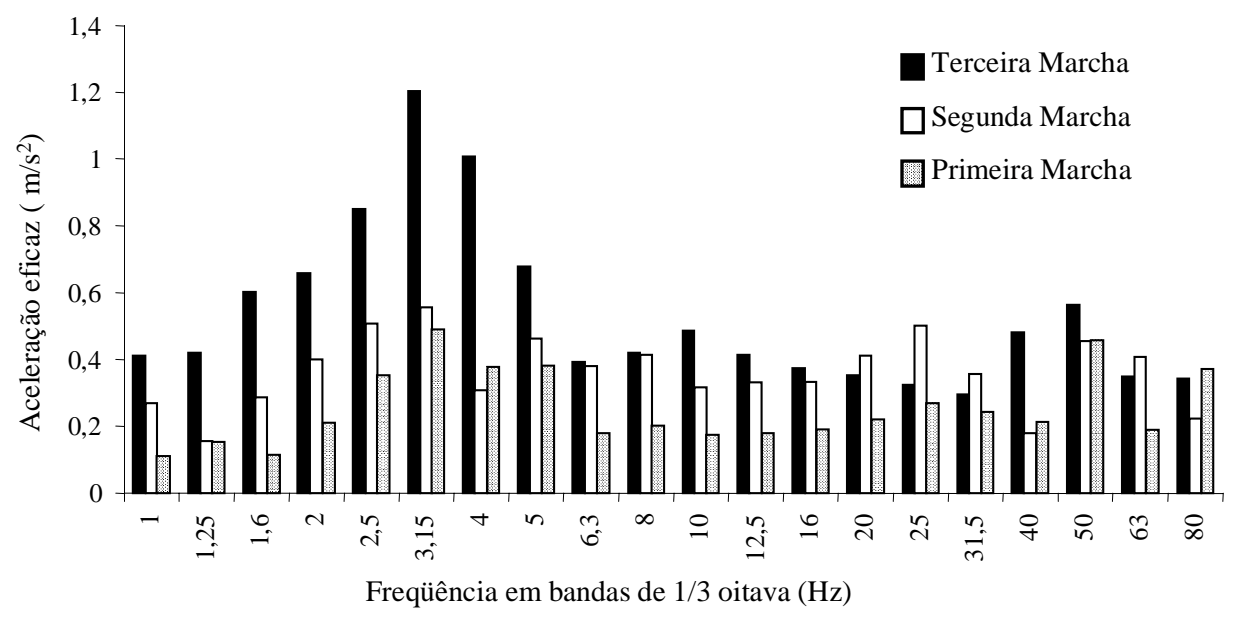

Figura 5 - Níveis de aceleração eficaz vertical em bandas de 1/3 oitava.

Figure 5 - Effective vertical acceleration levels in 1/ 3 octave bands.

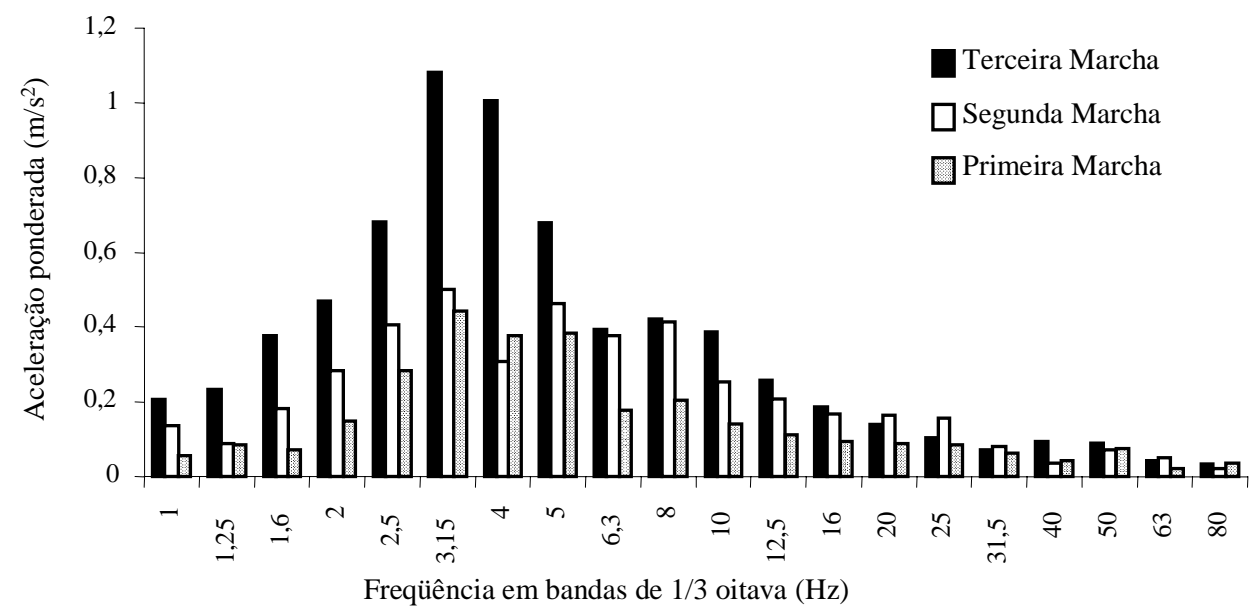

Figura 6 - Níveis de aceleração eficaz ponderada vertical em bandas del/3 oitava.

Figure 6 - Levels of effective weighted acceleration in 1/ 3 octave bands. 


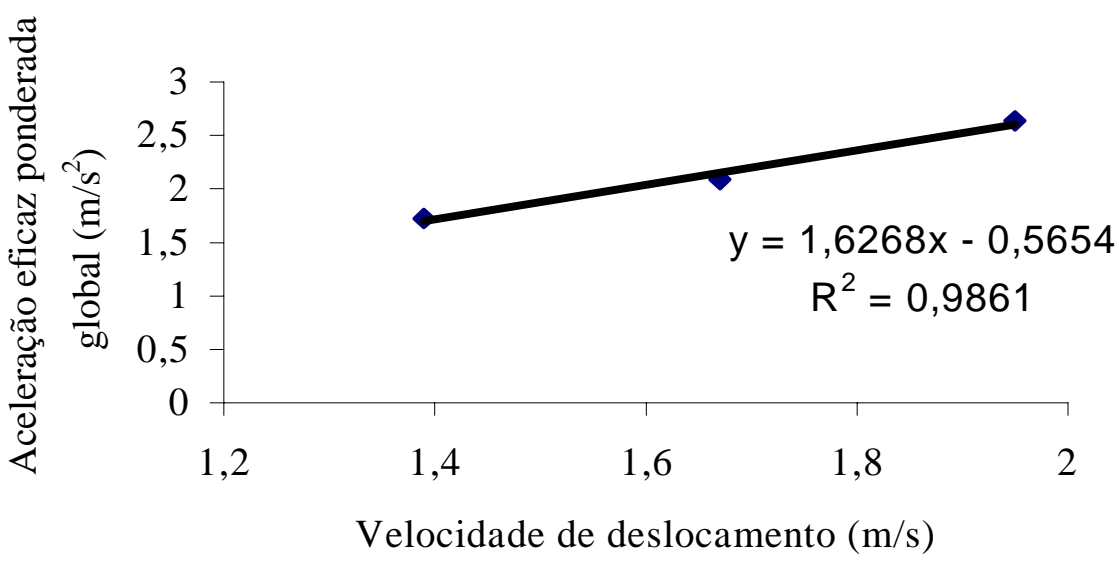

Figura 7 - Aceleração eficaz ponderada global para as três velocidades em estudo. Figure 7 - Effective accel eration meditated gl obal for the three speeds in study.

A maior aceleração eficaz em bandas de $1 / 3$ de oitava encontrada foi de $1,2 \mathrm{~m} / \mathrm{s}^{2}$, para terceira marcha, na freqüência de centro $3,15 \mathrm{~Hz}$, enquanto para a segunda e primeira marchas os valores foram $0,6 \mathrm{~m} / \mathrm{s}^{2}$ e $0,5 \mathrm{~m} / \mathrm{s}^{2}$, respectivamente. Observou-se uma atenuação dos níveis de aceleração vertical na faixa considerada crítica entre 4 e $8 \mathrm{~Hz}$, para todas as velocidades, o que ressalta a importância do sistema de suspensão do assento (Figuras 5 e 6). Todas as velocidades estudadas apresentaram o maior pico de aceleração na mesma banda de freqüência, caracterizando a independência da freqüência dominante em relação à variação da velocidade.

Estudos similares sobre a vibração em tratores, em diferentes terrenos e condições operacionais, caracterizaram a aceleração rms vertical como sendo a mais significativa. Matthews (1966) analisou os níveis de aceleração em um trator lastrado, movimentando-se sobre pastagem, e encontrou acelerações de $3 \mathrm{~m} / \mathrm{s}^{2}$ (vertical), $1,2 \mathrm{~m} / \mathrm{s}^{2}$ (longitudinal) e $2,0 \mathrm{~m} / \mathrm{s}^{2}$ (lateral).

Mehta et al. (2000) citados por Santos Filho (2002) coletaram vários valores de aceleração vertical, longitudinal e lateral no assento do trator, em diferentes operações agrícolas, comparando com a norma ISO 2631-1 (1985), e observaram que os maiores níveis de aceleração para cada implemento encontram-se no eixo vertical.

Os valores de aceleração ponderada global calculados encontram-se bem acima dos limites estabelecidos para uma exposição durante 4 horas de trabalho. Para a terceira marcha a aceleração ponderada global foi $2,638 \mathrm{~m} / \mathrm{s}^{2}$, enquanto para a segunda marcha foi $2,089 \mathrm{~m} / \mathrm{s}^{2}$ e para a primeira foi $1,727 \mathrm{~m} / \mathrm{s}^{2}$ (Figura 7 ).

\section{CONCLUSÕES}

De acordo com os resultados experimentais obtidos, verificou-se que:

- Os níveis de aceleração encontrados na parte superior foram consideravelmente inferiores àqueles encontrados na base, para as três velocidades testadas. Foram encontradas acelerações instantâneas de até $20 \mathrm{~m} / \mathrm{s}^{2}$ na base do assento, enquanto para o assento o máximo foi $2,5 \mathrm{~m} / \mathrm{s}^{2}$, o que mostra a eficiência de amortecimento do assento.

- Os maiores picos de vibração vertical no assento do operador, para a operação de gradagem, foram encontrados na faixa entre 2 e $4 \mathrm{~Hz}$, dada pela densidade espectral de potência para as três marchas avaliadas.

- Os valores de aceleração ponderada global calculados encontram-se bem acima dos limites determinados para uma exposição durante 4 horas de trabalho, de acordo com a norma ISO 2631 (1997). Para a terceira marcha a aceleração ponderada global foi de 2,638 m/ $\mathrm{s}^{2}$, enquanto para a segunda marcha foi $2,089 \mathrm{~m} / \mathrm{s}^{2}$ e para a primeira marcha foi $1,727 \mathrm{~m} / \mathrm{s}^{2}$. 


\section{REFERÊNCIAS BIBLIOGRÁFICAS}

BARGER, E. L. et al. Tratores e seus motores. (Traduzido por V. L.Shilling). New York: Edgard Blucher, 1963. 398 p.

BERASATEGUI, M. B. R. Modelización y simulación del comportamiento de um sistema mecánico com suspensión aplicado a los asientos de los tractores agrícolas. 2000. 264 f. Tese (Doutorado em Agronomía) - Universidad Politécnica de Madrid, Madrid, 2000.

DUL, J.; WEERDMEESTER, B. Ergonomia prática. (Traduzido por Itiro Iida). São Paulo: Edgard Blucher, 1995. $147 \mathrm{p}$.

MATHIAS, M. H. Análise de vibrações em tratores. 1989. 110 f. Dissertação (Mestrado em Engenharia Mecânica) Universidade Estadual Paulista, Guaratinguetá, 1989.

MATTHEWS, J. Ride comfort for tractor operators: IV Assesssment of ride quality of seats. Journal Agricultural Engineering Research, v. 11, p. 47-57, 1966.
MEHTA, C. R.; TEWARI, V. K. Seating discomfort for tractor operators-a critical review. International Journal of Industrial Ergonomics. v. 25, p. 661-674, 2000.

NAGAOKA, A. K. Desenvolvimento e avaliação do desempenho de um equipamento para ensaio dinâmico de rodado agrícola individual. 2001. 206 f. Tese (Doutorado em Energia na Agricultura) - Universidade Estadual Paulista, 2001.

SANTOS FILHO, P. F. Avaliação dos níveis de ruído e vibração vertical no assento de um trator agrícola de pneus utilizando um sistema de aquisição automática de dados. 2002. 53 f. Dissertação (Mestrado em Engenharia Agrícola) - Universidade Federal de Viçosa, Viçosa, 2002.

SCHLOSSER, J. F.; DEBIASI, H. Caderno técnico da Revista cultivar máquinas. edição janeiro/fevereiro 2002 (Conforto, preocupação com o operador). p. 3-9.

SMITH, L. A.; THOMAS JR., R. E. Investigaciones sobre ergonomia em el sudeste de los Estados Unidos. Unasylva, v. 44, p. 38-44, 1993. 